\title{
La leptina agisce indipendentemente dall'alimentazione nel modulare la composizione microbica dell'intestino in topi maschi
}

\author{
Marco Centanni · Maria Giulia Santaguida
}

Pubblicato online: 10 luglio 2014

(C) Springer International Publishing AG 2014

\section{Commento a:}

Leptin acts independently of food intake to modulate gut microbial composition in male mice.

M.W. Rajala, C.M. Patterson, J.S. Opp, S.K. Foltin, V.B. Young, M.G. Myers.

Endocrinology (2014) 155(3):748-757

La patogenesi di importanti disordini metabolici, quali l'obesità, la sindrome metabolica e le malattie cardiovascolari ad esse conseguenti, sembra essere significativamente influenzata dall'interazione dell'equilibrio dinamico esistente tra microbiota intestinale e ospite. La leptina, ormone di derivazione adipocitaria con effetto anoressizzante, agisce controllando l'appetito e il metabolic rate. L'obesità conseguente all'iperfagia e alla riduzione della spesa energetica nei topi $d b / d b$ e ob/ob è in relazione con l'inattivazione del recettore e/o del signaling della leptina. Questi animali presentano alterazioni peculiari del microbiota intestinale (incremento del rapporto Firmicutes/Bacteroidetes) simili a quelle degli animali in cui l'obesità è indotta dalla dieta. Il quesito cui lo studio di Rajala e coll. ha inteso rispondere è se sia proprio l'azione della leptina a modulare la composizione della popolazione batterica intestinale, in particolare analizzando l'espressione di peptidi antimicrobici intestinali (AMPs) prodotti e secreti nell'intestino dalle cellule di Paneth. Questi autori hanno perciò valutato l'espressione di diversi AMPs mediante PCR quantitativa su RNA estratto da cellule di ileo terminale ottenute da topi $d b / d b$ in alimentazione ad libitum e animali wild-type

M. Centanni $(\varangle) \cdot$ M.G. Santaguida

Dipartimento di Scienze e Biotecnologie Medico-Chirurgiche, UOC di Endocrinologia, "Sapienza" Università di Roma, Latina, Italia

e-mail: marco.centanni@uniroma1.it
(WT) di controllo. Il risultato di questo studio ha evidenziato una riduzione del $20-30 \%$ dell'espressione di specifici AMPs ( $\alpha$-defensina $1, \alpha$-defensina 5 e defcr-r) nei topi $d b / d b$. Questi peptidi, oltre a costituire una difesa nei confronti dei patogeni enterici, hanno anche la capacità di modulare la composizione dei microbi commensali, favorendo la colonizzazione di Bacteroidetes. Inoltre, stante il ruolo determinante del cibo nell'omeostasi microbica intestinale, è stato esaminato il profilo batterico in animali WT e topi $d b / d b$ con simile alimentazione e che non presentavano differenze nel peso corporeo. Il microbiota intestinale risultava comunque differente nei topi di controllo e nei $d b / d b$, nei quali l'espressione degli AMPs era significativamente ridotta. Questa evidenza suggerisce che la leptina agisca in maniera indipendente dall'alimentazione nel modulare l'espressione di questi peptidi e la composizione del microbiota. Quanto alla localizzazione dell'azione della leptina, mediante analisi immuno-istochimica, sono state esaminate le cellule che ne esprimono il recettore specifico (LepRb) a livello intestinale. Con questo approccio si è potuto valutare che l'azione della leptina si esplica non a livello dell'epitelio intestinale, ma su popolazioni cellulari a livello della sottomucosa in sede perivascolare. È stato inoltre evidenziato che l'espressione quantitativa del recettore in queste cellule aumenta progressivamente dalla porzione prossimale a quella distale dell'intestino ove raggiunge il massimo livello.

Si può quindi concludere che il signaling della leptina sia coinvolto nella regolazione della composizione del microbiota intestinale, a sua volta implicato nella genesi dell'obesità e della sindrome metabolica anche in maniera indipendente dall' alimentazione. 